\title{
ARTICLE OPEN \\ Genome-wide association study for proliferative diabetic retinopathy in Africans
}

\author{
Chang Liu ${ }^{1,2,3}$, Guanjie Chen (D) ${ }^{1}$, Amy R. Bentley (D) ${ }^{1}$, Ayo Doumatey ${ }^{1}$, Jie Zhou ${ }^{1}$, Adebowale Adeyemo (D) ${ }^{1}$, Jinkui Yang ${ }^{2,3}$ and \\ Charles Rotimi (iD)
}

Proliferative diabetic retinopathy (PDR) is a sight-threatening complication of diabetes that is associated with longer duration of diabetes and poor glycemic control under a genetic susceptibility background. Although GWAS of PDR have been conducted in Europeans and Asians, none has been done in continental Africans, a population at increased risk for PDR. Here, we report a GWAS of PDR among Africans. PDR cases $(n=64)$ were T2D patients with neovascularization in the retina and/or retinal detachment. Controls ( $n=227$ ) were T2D patients without listed eye complications despite high risk (T2D duration $\geq 10$ years and fasting blood glucose $>169 \mathrm{mg} / \mathrm{dl}$ ). Replication was assessed in African Americans enrolled in the ARIC study. We identified 4 significant loci: WDR72, HLA-B, GAP43/RP11-326J18.1, and AL713866.1. At WDR72 the most strongly associated SNPs were rs12906891 (MAF $=$ $0.071 ; p=9.68 \times 10-10 ; \mathrm{OR}=1.46,95 \% \mathrm{Cl}[1.30,1.64])$ and $\mathrm{rs} 11070992\left(\mathrm{MAF}=0.14 ; p=4.23 \times 10^{-8} ; \mathrm{OR}=1.28,95 \% \mathrm{Cl}[1.17-1.40]\right)$. rs11070992 replicated in African Americans $(p=0.04)$. Variants in this gene have been associated with diabetic retinopathy, glycemic control, revascularization, and kidney disease.

npj Genomic Medicine (2019)4:20 ; https://doi.org/10.1038/s41525-019-0094-7

\section{INTRODUCTION}

Diabetic retinopathy (DR) is a well-known complication of type 1 (T1D) and type 2 diabetes (T2D). It is the most frequent cause of new cases of blindness in the working-age population ${ }^{1}$ and the disorder progresses from non-proliferative DR (NPDR) to sightthreatening proliferative DR (PDR). The latter is characterized by the growth of abnormal new blood vessels on the retina. Neovascularization and contraction of the accompanying fibrous tissue can distort the retina and lead to tractional retinal detachment, producing severe and often irreversible vision loss. In addition, the new blood vessels may cause pre-retinal or vitreous hemorrhage and neovascular glaucoma, which can also cause vision loss. By 2020, the prevalence of DR is expected to almost double to 7.2 million within the USA alone. ${ }^{2}$ Notably, the prevalence of PDR varies considerably by ethnicity with estimates as high as $8.99 \%$ in Africans and 2.67, 1.29, and 5.10\%, respectively in Chinese, South Asian and Hispanic individuals ${ }^{3-7}$ African ancestry individuals within the US also display higher DR prevalence and more severe DR compared to non-Hispanic Whites. $^{8}$

Epidemiologic studies suggest that the severity of DR closely correlates with glycemic control and diabetes duration, ${ }^{2,9}$ and optimizing glycemic control is the main recommendation to reduce the risk or slow the progression of retinopathy. ${ }^{10}$ There is mounting evidence indicating a large genetic contribution to the development and severity of DR as well. ${ }^{11,12}$ Although the Family Investigation of Nephropathy and Diabetes (FIND)-Eye study reported a broad-sense heritability for DR of $27 \%$ overall, $^{13}$ estimates for PDR have been reported to be as high as $52 \%{ }^{14}$ Furthermore, even though those with T1D may have difficulty controlling their glycemia, many individuals living with T1D for 50 years or more never develop PDR. ${ }^{15,16}$ Together, these observations suggest that genetic factors may influence a diabetic individual's risk of developing DR and progression to PDR.

Knowledge about the specific genetic risk factors for DR has come from genome-wide association studies (GWAS) (Table S1) including studies of Taiwanese, Mexican-American, American (European Ancestry), Chinese, and Japanese T2D patients., 2,17-22 Despite African ancestry individuals displaying high prevalence of PDR, only one GWAS for PDR has been conducted in African Americans, and this study failed to identify any significantly associated loci. ${ }^{23}$

\section{RESULTS}

Characteristics of study participants are displayed in Table 1. Consistent with our selection criteria for controls, both duration of diabetes (AADM 15.5 years; ARIC-AA 17.3 years) and fasting blood glucose levels were high among controls (AADM $268.3 \mathrm{mg} / \mathrm{dl}$; ARIC-AA $283.7 \mathrm{mg} / \mathrm{dl}$ ). In AADM, cases also had higher triglycerides, HDL cholesterol and total cholesterol levels $(p<0.05)$. The effects of population stratification on our data were assessed through visualization of $Q-Q$ plots $(\lambda=1.015)$ using the full set of markers (Supplementary Fig. S1) and stratified by MAF (Supplementary Fig. S2). The effects were determined to be negligible following adjustment for the first two principal components. Relatively little additional information was found beyond the first 2 PCs, which are consistent with an East/West Africa split (PC1) and a gradient across West African samples (PC2) (Supplementary Fig. S3).

Association results for PDR showed 7 variants in 4 loci that reached genome-wide significance $\left(p<5 \times 10^{-8}\right)$ in the discovery

\footnotetext{
${ }^{1}$ The Center for Research on Genomics and Global Health, National Human Genome Research Institute, National Institutes of Health, Bethesda, MD, USA; ${ }^{2}$ Department of Endocrinology, Beijing Tongren Hospital, Capital Medical University, Beijing 10730, China and ${ }^{3}$ Beijing Diabetes institute, Beijing 100730, China Correspondence: Charles Rotimi (rotimic@mail.nih.gov)
}

Received: 18 January 2019 Accepted: 7 August 2019

Published online: 29 August 2019 
Table 1. Characteristics of study participants

\begin{tabular}{|c|c|c|c|c|c|c|}
\hline & \multicolumn{3}{|c|}{ Discovery (AADM) } & \multicolumn{3}{|c|}{ Replication (ARIC-AA) } \\
\hline $\mathrm{N}$ (\% in Female) & $64(57.81 \%)$ & $227(60.35 \%)$ & 0.8248 & $20(70.0 \%)$ & 59 (61.0\%) & 0.6514 \\
\hline Age (Years) & $59.89(10.10)$ & $58.08(9.67)$ & 0.2030 & $60.64(6.04)$ & $60.55(5.90)$ & 0.9522 \\
\hline SBP $(\mathrm{mmHg})$ & $147.5(27.68)$ & $140.4(24.71)$ & 0.0652 & $137.5(15.71)$ & $130.1(22.79)$ & 0.1135 \\
\hline $\mathrm{DBP}(\mathrm{mmHg})$ & $83.97(13.80)$ & $80.20(13.80)$ & 0.0568 & $75.3(12.92)$ & $76.45(11.07)$ & 0.7226 \\
\hline $\mathrm{HDL}-\mathrm{C}(\mathrm{mmol} / \mathrm{L})$ & $53.11(19.32)$ & $44.30(16.49)$ & 0.0015 & $52.90(14.7)$ & $48.14(13.35)$ & 0.2390 \\
\hline $\mathrm{CHOL}(\mathrm{mg} / \mathrm{dL})$ & $238.2(93.81)$ & $207.1(57.23)$ & 0.0151 & $229.1(54.60)$ & $204.3(37.22)$ & 0.4610 \\
\hline HTN Medication (\%) & $20(31.25 \%)$ & $100(44.05 \%)$ & $<0.0001$ & $13(65.0 \%)$ & $32(54.24 \%)$ & 0.4007 \\
\hline Lipid-lowering medication (\%) & $0(0 \%)$ & $2(0.88 \%)$ & 0.451 & $1(5.0 \%)$ & $2(3.39 \%)$ & 0.7448 \\
\hline LDL-C (mmol/L) & $142.8(52.60)$ & $139.0(46.98)$ & 0.6111 & $137.5(47.2)$ & $128.1(32.9)$ & 0.4610 \\
\hline
\end{tabular}

sample (Table 2; Fig. 1). Four of these SNPs were in intron 1 in WDR72, 1 SNP in HLA-B, 1 SNP in AL713866.1 and 1 in GAP43. The most statistically significant association was for the $T$ allele for rs12906891 $\left(p=9.68 \times 10^{-10}, \mathrm{OR}=1.46[1.30,1.64]\right)$ located in intron 1 of WDR72 on chromosome 15 . Two other WDR72 variants (rs11070992 and rs67619978), in linkage disequilibrium (LD) with rs12906891, which have the $r^{2}$ in AADM and ARICAA were 0.384/ 0.381 and $0.518 / 0.504$, respectively, were also genome-wide significant. In AADM the LD of rs11070992 and rs67619978 is high, $r^{2}=0.98$ and the $r^{2}$ of $r s 11070992$ and $r$ s3081219 is 0.87 . Population frequency data for rs3081219 is not available. Analysis conditioned on rs11070992 showed only one peak in this region (Fig. 2).

Replication in ARIC-AA was attempted for each of the variants that were genome-wide significant in the discovery sample of Africans. Of these, 2 were $p<0.05$ and in the same direction of association among African Americans, both of which were WDR72 variants (in linkage disequilibrium with each other $\left[r^{2}=0.9\right]$ ).

To further evaluate the variants that were genome-wide significant in the AADM analysis, we conducted several secondary analyses. To determine the extent to which our control selection enabled the detection of these loci, we also ran analyses using any type 2 diabetic participant without PDR as a control. For 6 of the 7 variants tested, the statistical significance was reduced, despite the larger sample size for the controls using this strategy (Table S2). The exception was rs72740408 (AL713866.1), which was more statistically significant using $\mathrm{T} 2 \mathrm{D}$ controls, though the effect was reduced (extreme: OR 1.86, $p=1.1 \mathrm{E}-8$; T2D: OR 1.15, $p=1.6 \mathrm{E}$ -11). We also tested the lead SNPs from the PDR association for an association with fasting blood glucose among AADM participants (stratified by case status, given the effect of medication on glucose values); one of the variants tested reached nominal significance ( $\mathrm{rs} 12906891$ in WDR72: beta $-0.07, p=0.03$; Table S3). Adding glucose as a covariate to the analysis of PDR slightly reduced the statistical significance of the results but does not appear to fully mediate the association with PDR ( $p$-values of lead SNPs in adjusted analysis: $6.2 \mathrm{E}-6$ to $3.3 \mathrm{E}-8$; Table S4).

The HLA-B variant that was genome-wide significant in the analysis of Africans (rs1065386), produces a missense change at position 87 of the amino acid sequence that is found on several classical HLA-B alleles, including HLA-B*07:02, HLA-B*07:05, HLA$B^{*} 14: 01$, and $H L A-B^{*} 35: 01$, among others. We tested the classical HLA-B alleles that include the rs1065386 variant allele for an association with PDR in our data (Table S5). HLA-B*07:02 ( $p=$ $0.016)$ and $\mathrm{HLA}-\mathrm{B}^{*} 45: 01 \quad(p=0.017)$ were nominally significantly associated with PDR in the AADM data.

We also investigated previously reported top associations for PDR in our data, though few of these associations reached genome-wide significance in the original publications (Supplementary Table S6). In both AADM and ARIC-AA, we replicated rs1902491 (OR $=0.91 / 0.85, \quad p=0.0013 / 0.02)$ near NPY2R/ LOC729902, which was reported in three different cohort studies ${ }^{2}$. In AADM, we replicated $\mathrm{rs} 918519(\mathrm{OR}=0.913, p=0.014)$ near LOC285626, which was recently reported in White Australian individuals. ${ }^{22}$ While our replication supports these previous findings, neither of these associations were GWAS significant in the original publication, and they would not reach this threshold with meta-analysis with our data.

\section{DISCUSSION}

Despite evidence for a genetic contribution to proliferative diabetic retinopathy, GWAS have not been very successful in identifying loci for PDR. Many of the results in previously published GWAS of PDR did not reach genome-wide significance (Supplementary Table S1), and the top loci from these studies have not been replicated in independent populations. $2,17,20,24,25$ Results from candidate gene association studies have also been conflicting. ${ }^{26}$ These studies vary considerably in case-control and outcome definition. In our study, we use an "extreme phenotype" approach. As diabetic retinopathy is closely related to glycemic level and diabetes duration, ${ }^{2,9}$ we chose controls who had at least 10 years T2D duration and high glycemia but with no evidence of DR. By selecting controls that had sufficient environmental risk to make them susceptible to PDR, we decreased the chance that any of the controls had genetic risk factors for PDR: under these conditions, individuals with predisposing genetic factors for PDR would be expected to have developed it. Based on this careful definition of controls, this first GWAS of PDR in continental 


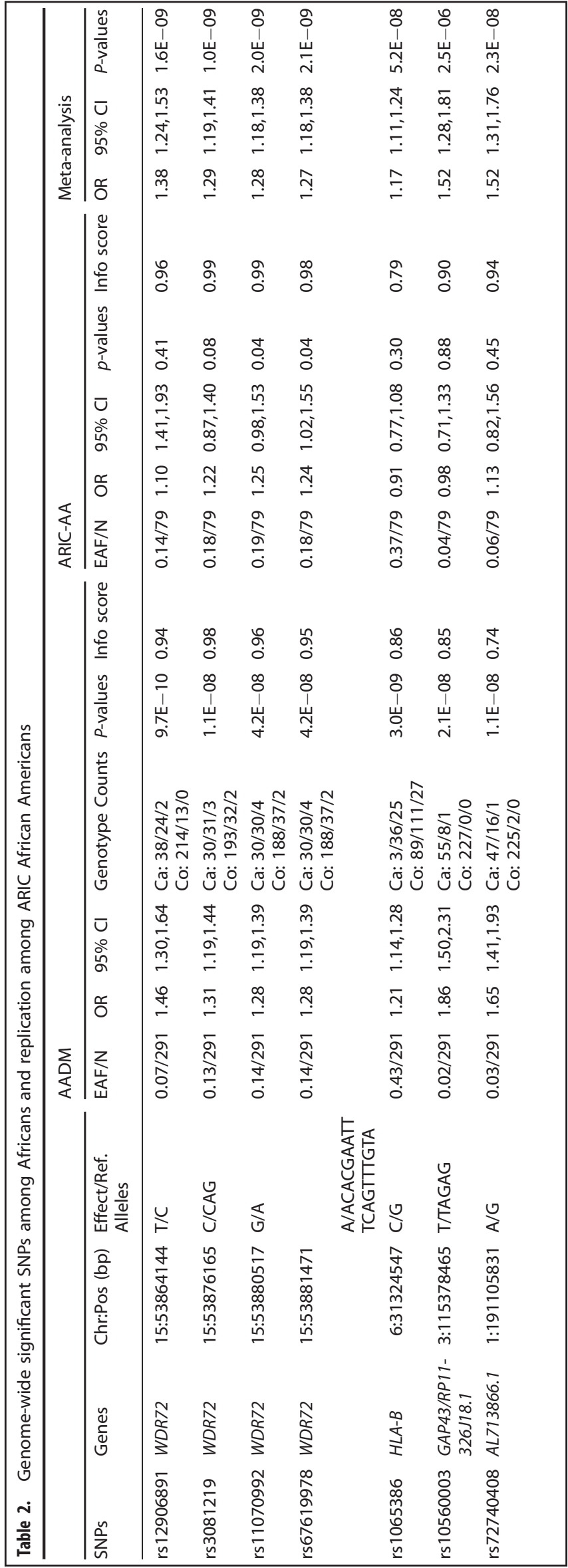

Africans with T2D identified 4 genome-wide significant loci with replication of one of these (WDR72) in African Americans. ${ }^{27}$

WDR72 (WD repeat-containing protein $72,15 q 21.3$ ) is a proteincoding gene that has been associated with $\mathrm{HbA} 1 \mathrm{c}$ level and poor glucose control. ${ }^{28}$ Notably, WDR72 mRNA is expressed in the retina. $^{28}$ Diabetic kidney disease (DKD) and DR are both microvascular complications of T2D and share pathophysiological mechanisms and common risk factors. ${ }^{25}$ Some DKD studies require the presence of $D R$ in controls, highlighting the correlation between these outcomes. ${ }^{25}$ WDR72 is associated with eGFR and is highly expressed in the kidney epithelium, suggesting that genetic variation at these loci may have relevance for both microvascular complications. $^{28,29}$ An important paralog of WDR72, WDR7, is associated with retinitis pigmentosa 4 , a highly expressed protein in the retina (https://www.genecards.org/cgi-bin/carddisp.pl? gene $=$ WDR7). Additionally, WDR72 is critical to ameloblast vesicle turnover during enamel maturation. ${ }^{30}$ Enamel matrix derivative induces proliferation, viability, and angiogenesis of human microvascular cells. ${ }^{31}$ These observations suggest WDR72 may be involved in proliferative angiogenesis of the microvasculature in the eyes.

HLA-B (Major Histocompatibility complex class 1, B) is a proteincoding gene which is expressed in the retina and the pancreas. HLA-B was also associated with DR in African and Caucasian in T1DM. $^{32,33}$ In terms of classical HLA alleles, the HLA-B alleles showing significant association with PDR in the present study are HLA-B*07:02 $(p=0.0155)$ and HLA-B*45:01 $(p=0.0171)$. These are not the same classical HLA-B alleles reportedly associated with DR in T1DM: HLA-B49 ${ }^{32}$ and HLA-B62. ${ }^{33}$

GAP43 (growth-associated protein 43, gene ID:172020, 3q13.31) is a protein-coding gene which is expressed in the retina and pancreas. GAP43 is also an axonal regenerative marker which is related to diabetic peripheral neuropathy, while axons and blood vessels share molecular signals for purpose of navigation, regeneration, and terminal arborizations. ${ }^{34}$ The function of rs72740408 located near RNA gene $A L 713866.1$ is still unknown.

We also replicated previous reported PDR associations with rs1902491 (near NYP2R) and rs918519 (near LOC285626). Neuropeptide $\mathrm{Y}$ Receptor subtype 2(NPY2R) is a G protein-coupled receptor for NPY, a neurotransmitter released by endothelial cells implicated in ischemic angiogenesis. ${ }^{35}$ There is substantial genetic, biologic, and functional data supporting a role for neuropeptide $Y$ signaling in diabetic retinopathy. ${ }^{36-39}$ rs918519 (35 kb upstream of LOC285626) has been previously associated with PDR. ${ }^{22}$ This gene encodes an uncharacterized, long noncoding RNA. The nearest protein-coding gene is IL12B (Interleukin $12 B)$, a further $33 \mathrm{~kb}$ upstream. IL12B is expressed in the retina (The Ocular Tissue Database ${ }^{40}$ ) and has been implicated in both $\mathrm{T}_{1 \mathrm{D}}{ }^{41-43}$ and T2D. ${ }^{44}$

To date, only one other study has used a similar control selection strategy (only including diabetics with a long duration of disease). In that study, Shtir et al. ${ }^{45}$ performed whole-exome sequencing on 64 cases and 43 controls of Saudi descent. They identified three genes, NME3, LOC728699, and FASTK, whose increased rare variant burden appeared to protect against DR. We did not find association in these genes in our study, which may reflect our focus on common variants.

A potential limitation of this study is the small sample size which is partly due to the strict definition of controls to include only those at high risk that did not develop PDR; however, we believed this approach which resulted in "cleaner phenotypic classification" enhanced our ability to identify GWAS significant loci for PDR. When we evaluated the identified associations in an analysis that used all diabetics as controls instead of our extreme control design, we see a diluted association for all but one variant, consistent with the less carefully defined controls adding uncertainty to the analysis, as some of the controls may have become cases with sufficient length of T2D diagnosis or poor 


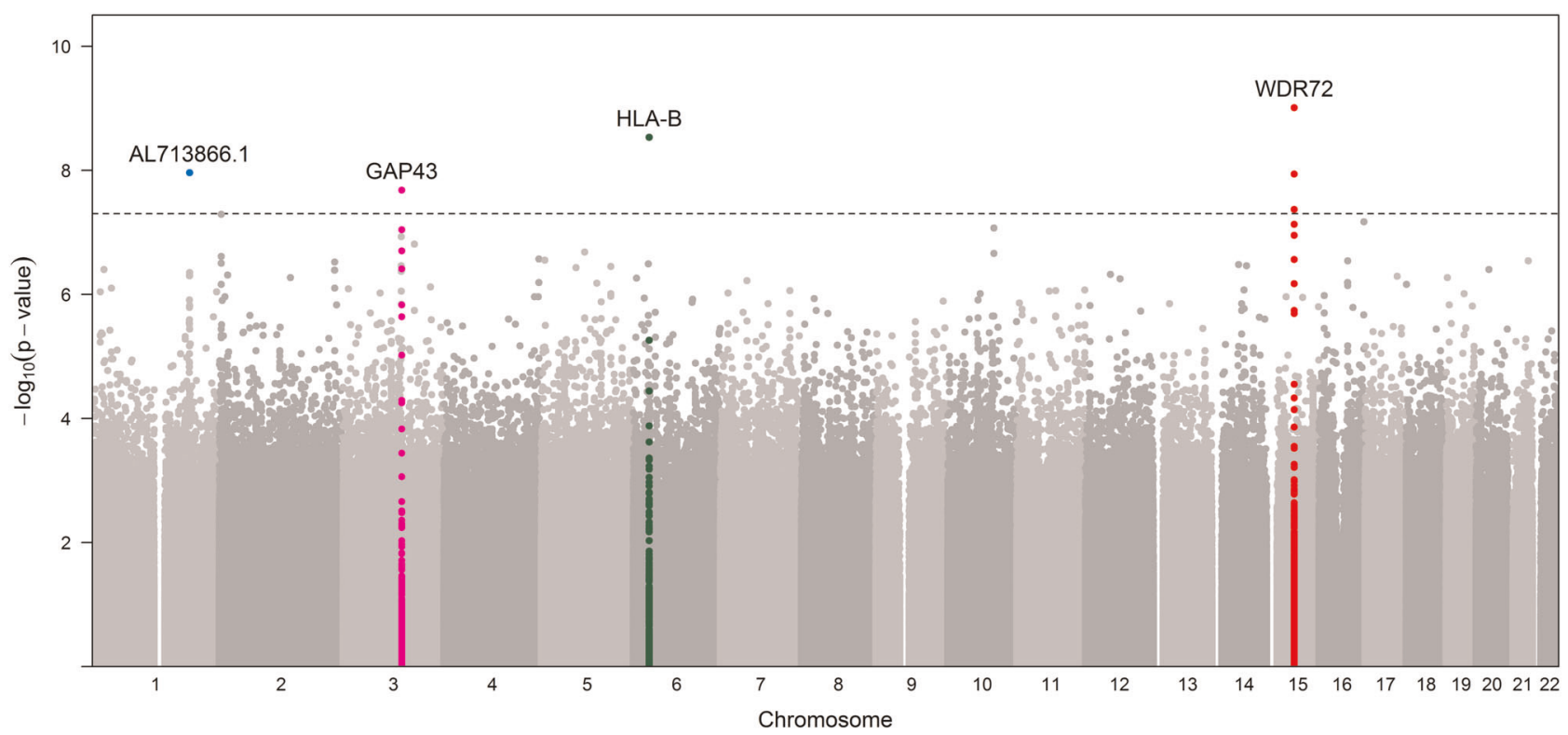

Fig. 1 GWAS Manhattan plot for PDR in the AADM study. The dotted line represents $-\log 10\left(5 \times 10^{-8}\right)$

a

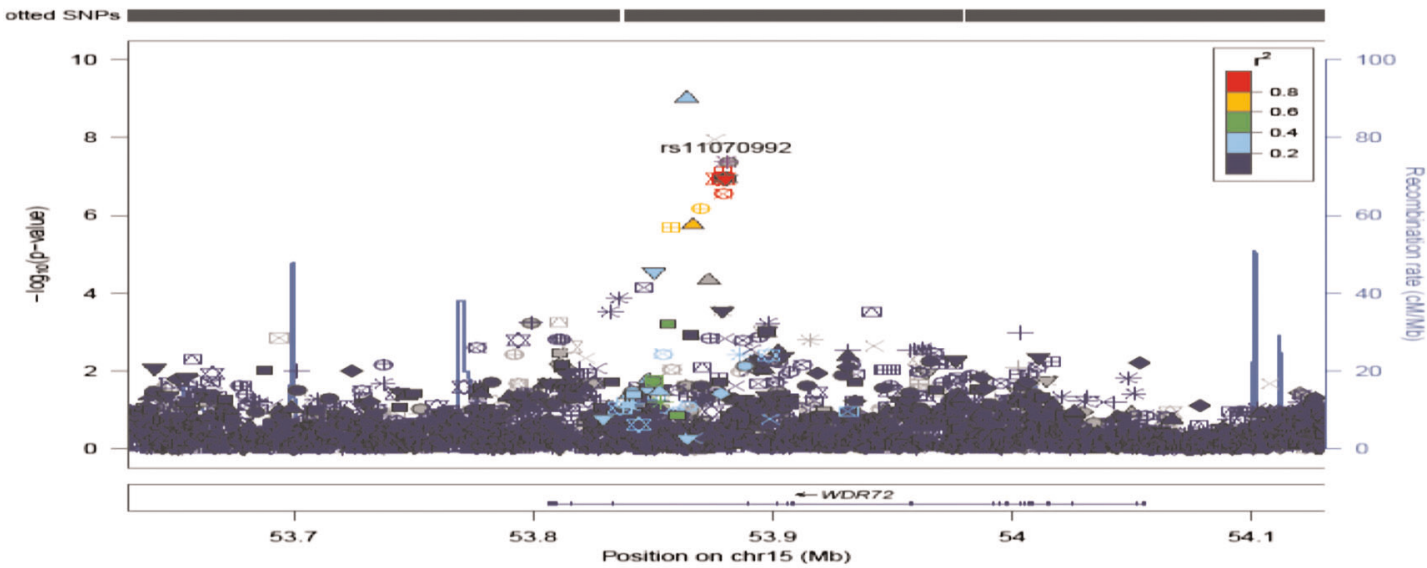

b

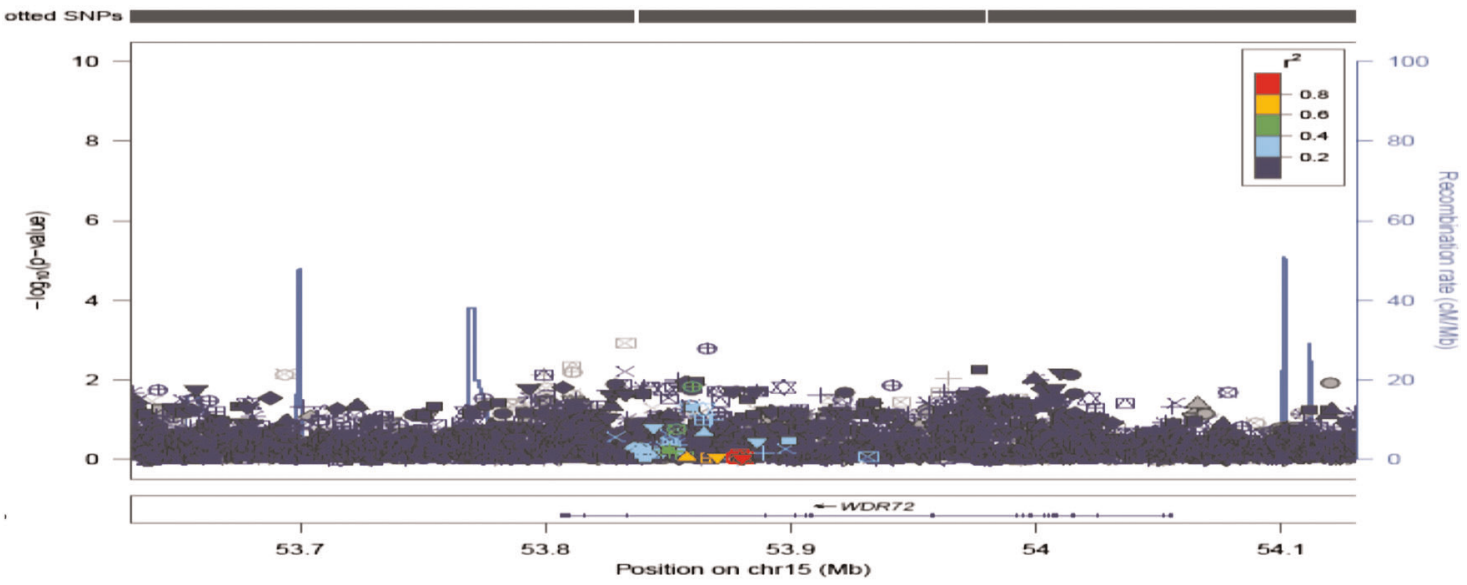

Fig. 2 Regional plots for association and conditional analyses on rs 11070992 in WDR72, using AFR from 1000 Genomes data ${ }^{60}$ as an LD reference. On the left $y$-axis is the -log10( $p$-value) for each SNP genotyped. On the right $y$-axis are the recombination rates estimated from the 1000 genome project. The annotated genes in the region are indicated along the bottom of the figure. Panels show the regional associations before (a) and after (b) adjusting for rs11070992 
glucose control. It is anticipated that a greater number of subjects, using this strategy to define control subjects, will yield even more loci. Our discovery sample consisted of continental Africans, while our replication set was African Americans. While it would have been ideal to conduct replication in Africans, as well, we are unaware of other datasets of Africans in which such an analysis could be conducted. Thus, it is uncertain whether our inability to replicate some of our findings was a result of false positives or other factors including small replication sample size, or differences in genomic (e.g., admixture) or environmental context between Africans and African Americans.

In all, this GWAS of PDR among Africans identified 4 genomewide significant loci, one of which replicated among African Americans. Identified loci highlight pathways of known significance to diabetic retinopathy, including glycemic control, and revascularization.

\section{METHODS}

Ethics statement

The AADM study was approved by the Institutional Review Board (IRB) at the National Institutes of Health, and the ARIC study was approved by the IRB of all participating institutions, including the IRBs of the University of Minnesota, Johns Hopkins University, University of North Carolina, University of Mississippi Medical Center, and Wake Forest University. Both studies were conducted in accordance with the Declaration of Helsinki. Written informed consent was obtained from all human participants.

\section{Study design}

Study individuals were drawn from the AADM study, a large, ongoing genetic epidemiology study of T2D and related traits in Africans. ${ }^{46}$ Demographic information was collected using standardized questionnaires across the AADM study centers. Anthropometric, medical history, and clinical examination parameters were obtained by trained study staff during a clinic visit. Blood samples were drawn after an overnight fast of at least $8 \mathrm{~h}$. The definition of T2D was based on the American Diabetes Association (ADA) criteria. ${ }^{47}$ Biochemical parameters were measured with a Beckman-UniCel Dxc 800 biochemistry analyzer (Beckman Coulter, Carlsbad, (A), including fasting plasma glucose, total cholesterol, triglycerides, LDL and HDL cholesterol.

\section{Evaluation of PDR}

All AADM study participants underwent a thorough ophthalmological examination. The eye examination was part of a comprehensive physical examination of each participant in the study. Each participant had the following ocular examinations: visual acuity; ocular alignment and motility; pupil reactivity and function; visual fields; intraocular pressure; slit lamp examination of the cornea, iris, lens, and vitreous; and dilated fundus examination. Previous experiences of the ophthalmologists in the 5 centers demonstrate a better than $80 \%$ concurrence between their clinical and photographic assessment of the presence and absence of lesions associated with diabetic retinopathy. To assure reproducibility of the assessment and classification of ocular complications between the 5 centers, the absence and/or presence of hemorrhages, microaneurysms, cotton wool spots, neovascularization, cataracts, retinal detachment, maculopathy, and glaucoma in each subject's eyes was recorded, along with other ocular abnormalities. ${ }^{48} \mathrm{~A}$ diagnosis of PDR was made only with neovascularization in any field or retinal detachment with a diagnosis of diabetes retinopathy.

\section{Selection of PDR cases and controls}

Patients with PDR (neovascularization and or retinal detachment) were selected as cases, irrespective of glucose level and duration of diabetes.

While most genetic studies of PDR to date have used a general sample of T2D patients as the control group, this approach runs the risk of misclassifying T2D patients without a long history of disease or with good glycemic control as controls as they might develop disease if challenged with poor glucose control or with a longer time since diagnosis. To minimize such misclassification bias, we selected as controls only those patients with fasting blood glucose of at least $169 \mathrm{mg} / \mathrm{dl}$ (which is equivalent to $\mathrm{HbA} 1 \mathrm{C}>7.5 \%[58 \mathrm{mmol} / \mathrm{mol}])$ and with a duration of diabetes of at least 10 years but without diabetic retinopathy (NDR). We reasoned that using such "super-controls" will improve the power of the study through more precise phenotype definition, albeit at the cost of a reduction in sample size.

\section{Genotyping and imputation}

A total of 5231 African individuals from the AADM study were genotyped on high density GWAS arrays (Affymetrix ${ }^{\circledR}$ Axiom ${ }^{\circledR}$ Genome-Wide PanAFR and Illumina Multi-Ethnic Genotyping arrays. Comparable proportions of cases were genotyped on each array (Affymetrix: 22.9\%, Illumina: $21.1 \%$ ).

After technical quality control and appropriate sample- and SNP-level filtering (individual call rate $\leq 95 \%$, SNP call rates $\leq 95 \%$, Hardy-Weinberg $p$ $<10-6$, and minor allele frequency $(\mathrm{MAF})<0.01)$, imputation was performed using the African Genome Resources Panel available from the Sanger Imputation Service (https://imputation.sanger.ac.uk/). ${ }^{49}$ The African Genome Resources Imputation Reference Panel comprised 4956 individuals, including all 2504 from the 1000 Genomes Project Phase 3, 2000 individuals from Uganda (Baganda, Banyarwanda, Barundi, and others), and $~ 100$ individuals from each of a set of populations from Ethiopia (Gumuz, Wolayta, Amhara, Oromo, and Somali), Egypt, Namibia (Nama/ Khoe-San) and South Africa (Zulu), yielding 9912 haplotypes for 93,421,145 SNPs. Pre-phasing was performed with EAGLE version $2.0 .5^{50}$ and imputation was performed using software Positional Burrows-Wheeler Transform (PBWT). ${ }^{51}$ The resulting imputation dataset was filtered for $M A F \geq 0.01$ and info $\geq 0.3$. A total of $18,433,741$ SNPs was tested. Coordinates are given based on the hg19 genome build.

\section{Association analysis}

Association analysis was performed using the EPACTS 3.2.6 (Efficient and Parallelizable Association Container Toolbox) pipeline (http://genome.sph. umich.edu/wiki/EPACTS), using imputed dosages and adjusting for genetic relatedness, sex, age, and the first two principal components. Within EPACTS, we performed single variant EMMAX association analysis. Genome-wide significance was declared to be $p<5 \times 10^{-8}$. Q-Q and Manhattan plots were generated using R 3.4 .2 (http://www.r-project.org).

Secondary analyses were conducted to further characterize the top SNPs of our main analysis (all $p<5 \times 10^{-8}$ ). To evaluate our control selection strategy, we also ran analyses as above, but included as controls all AADM participants with T2D, but no PDR, regardless of duration of T2D or presence of hyperglycemia $(n=1724)$. To determine whether these variants were associated with PDR through an association with glucose, we also ran our main analysis model with the addition of glucose as a covariate. Additionally, we tested these variants for an association with glucose, separately in AADM participants with $(n=2048)$ and without T2D ( $n=2133$; to avoid effects of treatment on glucose concentration). We further evaluated a finding in the HLA-B region by conduct HLA imputation using the HIBAG algorithm ${ }^{52}$ and show that the missense change is on several classic HLA-B alleles.

\section{Replication study and meta-analysis}

The replication study was conducted in the Atherosclerosis Risk in Communities (ARIC) study accessed through dbGaP (phs000280.v2. p1). ARIC was designed as a multi-ethnic study of atherosclerosis and recruited individuals in Forsyth County, NC; Jackson, MS; the suburbs of Minneapolis, MN; and Washington County, MD. It is a population-based study of 15,792 individuals aged 45-64 years at their first examined in 1987-1989. ${ }^{53} \mathrm{~A}$ second examination was conducted in 1990-1992 and a third in 1993-1995 when retinal photographs were taken on all participants. ${ }^{54}$ Our analysis only includes AAs of the third phase of ARIC. One randomly selected eye was photographed using a nonmydriatic camera and evaluated by masked graders according to standardized protocols, as has previously been described, ${ }^{54,55}$ allowing for consistent PDR definitions with AADM. Individuals from ARIC were genotyped using the Affymetrix ${ }^{\circledast}$ Genome-Wide SNP Array 6.0.53 For the ARIC data, imputation was performed using impute2 software and 1000 genome phase 3 panel from the Sanger imputation service (https://imputation.sanger.ac.uk/). The resulting imputation dataset was filtered for $M A F \geq 0.01$ and info $\geq 0.3$.

Cases and controls were defined as in the discovery study. Replication was assessed in 19 cases and 50 controls obtained from the ARIC African Americans study (ARIC-AA; $n=3137$ ). ${ }^{56}$ Imputation and association analysis were performed as described above. We adjusted for genetic relatedness, sex, age, and the first two principal components. METAL ${ }^{57}$ was 
used to perform inverse variance-weighted fixed-effect meta-analysis of the discovery and replication samples.

Regional plot and conditional analysis

Association results for PDR at a 250-kb region surrounding rs11070992 (WDR72) were plotted using Locus Zoom (http://www.locuszoom.org). Conditional analysis was conducted using PLINK 1.9.58

\section{Statistical analysis}

Demographic and clinical characteristics were compared using chi-squared tests for dichotomous traits and t-tests for continuous traits. R-studio was used for preparation of the phenotype data and annotation.

To minimize the potential effect of population structure, we adjusted all analyses by principal components obtained from the $\mathrm{R}$ package SNPRelate, ${ }^{59}$ which uses genotyped SNPs to generate a genetic covariance matrix followed by eigen decomposition.

\section{Reporting summary}

Further information on research design is available in the Nature Research Reporting Summary linked to this article.

\section{DATA AVAILABILITY}

The datasets generated during and/or analyzed during the current study are not publicly available due to the inconsistency of study consent documents with repository deposition but are available from the corresponding author upon reasonable request.

\section{ACKNOWLEDGEMENTS}

The contents of this publication are solely the responsibility of the authors and do not necessarily represent the official view of the National Institutes of Health. This research was supported by the Intramural Research Program of the Center for Research on Genomics and Global Health (CRGGH). The CRGGH is supported by the National Human Genome Research Institute, the National Institute of Diabetes and Digestive and Kidney Diseases, the Center for Information Technology, and the Office of the Director at the National Institutes of Health (1ZIAHG200362). This work utilized the computational resources of the NIH HPC Biowulf cluster (https://hpc.nih.gov).

\section{AUTHOR CONTRIBUTIONS}

Conceptualization: C.R., G.C., A.B., J.Y., and C.L. Data curation: J.Z. Formal analysis: G.C., C.L., and A.B. Funding acquisition: C.R. Investigation: G.C., A.B., A.A., and C.L. Methodology: G.C., A.A., C.R., A.D., and, A.B. Supervision: C.R. Writing: original draft: C. L. A.B. A.A. and C.R. Writing: review and editing: C.L. A.B. G.C. A.A., and C.R.

\section{ADDITIONAL INFORMATION}

Supplementary Information accompanies the paper on the npj Genomic Medicine website (https://doi.org/10.1038/s41525-019-0094-7).

Competing interests: The authors declare no competing interests.

Publisher's note: Springer Nature remains neutral with regard to jurisdictional claims in published maps and institutional affiliations.

\section{REFERENCES}

1. Fong, D. S. et al. Retinopathy in diabetes. Diabetes Care 27, S84-87 (2004).

2. Grassi, M. A. et al. Genome-wide meta-analysis for severe diabetic retinopathy. Hum. Mol. Genet 20, 2472-2481 (2011).

3. Smith, T. S., Szetu, J. \& Bourne, R. R. The prevalence and severity of diabetic retinopathy, associated risk factors and vision loss in patients registered with type 2 diabetes in Luganville, Vanuatu. Br. J. Ophthalmol. 91, 415-419 (2007).

4. Wang, F. H. et al. Prevalence of diabetic retinopathy in rural China: the Handan Eye Study. Ophthalmology 116, 461-467 (2009).

5. Chetthakul, T. et al. Thailand diabetes registry project: prevalence of diabetic retinopathy and associated factors in type 2 diabetes mellitus. J. Med Assoc. Thai 89, S27-36 (2006)
6. Wang, N. et al. The status of diabetic retinopathy and diabetic macular edema in patients with type 2 diabetes: a survey from Beixinjing District of Shanghai city in China. Ophthalmologica 222, 32-36 (2008).

7. Yau, J. W. et al. Global prevalence and major risk factors of diabetic retinopathy. Diabetes Care 35, 556-564 (2012).

8. Emanuele, N. et al. Ethnicity, race, and baseline retinopathy correlates in the veterans affairs diabetes trial. Diabetes Care 28, 1954-1958 (2005).

9. Klein, R., Klein, B. E., Moss, S. E., Davis, M. D. \& DeMets, D. L. The Wisconsin epidemiologic study of diabetic retinopathy. IV. Diabet. macular edema. Ophthalmol. 91, 1464-1474 (1984).

10. American Diabetes, A. Standards of medical care in diabetes-2011. Diabetes Care 34, S11-61 (2011).

11. Hallman, D. M. et al. Familial aggregation of severity of diabetic retinopathy in Mexican Americans from Starr County, Texas. Diabetes Care 28, 1163-1168 (2005).

12. Clustering of long-term complications in families with diabetes in the diabetes control and complications trial. The Diabetes Control and Complications Trial Research Group. Diabetes 46, 1829-1839 (1997).

13. Arar, N. H. et al. Heritability of the severity of diabetic retinopathy: the FIND-Eye study. Invest Ophthalmol. Vis. Sci. 49, 3839-3845 (2008).

14. Hietala, K., Forsblom, C., Summanen, P., Groop, P. H. \& FinnDiane Study, G. Heritability of proliferative diabetic retinopathy. Diabetes 57, 2176-2180 (2008).

15. Sun, J. K. et al. Protection from retinopathy and other complications in patients with type 1 diabetes of extreme duration: the joslin 50-year medalist study. Diabetes Care 34, 968-974 (2011).

16. Conway, B. N., Maynard, J. D. \& Orchard, T. J. Comment on: Sun et al. Protection from retinopathy and other complications in patients with type 1 diabetes of extreme duration: the Joslin 50-Year Medalist Study. Diabetes Care 2011;34:968-974. Diabetes Care 34, e148; author reply e149, https://doi.org/ 10.2337/dc11-0971 (2011)

17. Huang, Y. C. et al. Genome-wide association study of diabetic retinopathy in a Taiwanese population. Ophthalmology 118, 642-648 (2011).

18. Fu, W. et al. Analysis of 6,515 exomes reveals the recent origin of most human protein-coding variants. Nature 493, 216-220 (2013).

19. Awata, T. et al. A genome-wide association study for diabetic retinopathy in a Japanese population: potential association with a long intergenic non-coding RNA. PLOS ONE 9, e111715 (2014).

20. Sheu, W. H. et al. Genome-wide association study in a Chinese population with diabetic retinopathy. Hum. Mol. Genet 22, 3165-3173 (2013).

21. Burdon, K. P. et al. Genome-wide association study for sight-threatening diabetic retinopathy reveals association with genetic variation near the GRB2 gene. Diabetologia 58, 2288-2297 (2015).

22. Graham, P. S. et al. Genome-wide association studies for diabetic macular edema and proliferative diabetic retinopathy. BMC Med Genet 19, 71 (2018).

23. Pollack, S. et al. Multiethnic Genome-Wide Association Study of Diabetic Retinopathy Using Liability Threshold Modeling of Duration of Diabetes and Glycemic Control. Diabetes 68, 441-456 (2019).

24. Fu, Y. P. et al. Identification of diabetic retinopathy genes through a genomewide association study among Mexican-Americans from Starr County, Texas. J. Ophthalmol. 2010, https://doi.org/10.1155/2010/861291 (2010).

25. Hosseini, S. M. et al. The association of previously reported polymorphisms for microvascular complications in a meta-analysis of diabetic retinopathy. Hum. Genet 134, 247-257 (2015).

26. Abhary, S., Hewitt, A. W., Burdon, K. P. \& Craig, J. E. A systematic meta-analysis of genetic association studies for diabetic retinopathy. Diabetes 58, 2137-2147 (2009).

27. Tandon, A. et al. African ancestry analysis and admixture genetic mapping for proliferative diabetic retinopathy in African Americans. Invest Ophthalmol. Vis. Sci. 56, 3999-4005 (2015).

28. Paterson, A. D. et al. A genome-wide association study identifies a novel major locus for glycemic control in type 1 diabetes, as measured by both $\mathrm{A} 1 \mathrm{C}$ and glucose. Diabetes 59, 539-549 (2010).

29. Hertel, J. K. et al. Evaluation of four novel genetic variants affecting hemoglobin A1c levels in a population-based type 2 diabetes cohort (the HUNT2 study). BMC Med. Genet. 12, 20 (2011).

30. El-Sayed, W. et al. Mutations in the beta propeller WDR72 cause autosomalrecessive hypomaturation amelogenesis imperfecta. Am. J. Hum. Genet 85, 699-705 (2009).

31. Aspriello, S. D. et al. Effects of enamel matrix derivative on vascular endothelial growth factor expression and microvessel density in gingival tissues of periodontal pocket: a comparative study. J. Periodo. 82, 606-612 (2011).

32. Raache, R. et al. [Susceptibility genes, HLA and diabetic retinopathy in the Algerian population]. J. Fr. Ophtalmol. 36, 247-254 (2013) 
33. Mimura, T. et al. Relationship between human leukocyte antigen status and proliferative diabetic retinopathy in patients with younger-onset type 1 diabetes mellitus. Am. J. Ophthalmol. 135, 844-848 (2003).

34. Ebenezer, G. J. et al. Impaired neurovascular repair in subjects with diabetes following experimental intracutaneous axotomy. Brain 134, 1853-1863 (2011).

35. Zukowska, Z., Grant, D. S. \& Lee, E. W. Neuropeptide Y: a novel mechanism for ischemic angiogenesis. Trends Cardiovasc. Med. 13, 86-92 (2003).

36. Korn, J. M. et al. Integrated genotype calling and association analysis of SNPs, common copy number polymorphisms and rare CNVs. Nat. Genet 40, 1253-1260 (2008).

37. Price, A. L. et al. Principal components analysis corrects for stratification in genome-wide association studies. Nat. Genet 38, 904-909 (2006).

38. Pe'er, I., Yelensky, R., Altshuler, D. \& Daly, M. J. Estimation of the multiple testing burden for genomewide association studies of nearly all common variants. Genet Epidemiol. 32, 381-385 (2008).

39. Sandirasegarane, L. \& Kester, M. Enhanced stimulation of Akt-3/protein kinase Bgamma in human aortic smooth muscle cells. Biochem. Biophys. Res Commun. 283, 158-163 (2001).

40. Braun, T. A. et al. Non-exomic and synonymous variants in ABCA4 are an important cause of Stargardt disease. Hum. Mol. Genet. 22, 5136-5145 (2013).

41. Finkel, T. H. et al. Variants in CXCR4 associate with juvenile idiopathic arthritis susceptibility. BMC Med. Genet. 17, 24 (2016).

42. Morahan, G. et al. Evaluation of IL12B as a candidate type I diabetes susceptibility gene using data from the Type I Diabetes Genetics Consortium. Genes Immun. 10, S64-68 (2009).

43. Li, J. et al. Relationship between the IL12B ( $r$ 3212227) gene polymorphism and susceptibility to multiple autoimmune diseases: A meta-analysis. Mod. Rheuma. 26, 749-756 (2016)

44. Eiris, N. et al. Genetic variation at IL12B, IL23R and IL23A is associated with psoriasis severity, psoriatic arthritis and type 2 diabetes mellitus. J. Dermatol Sci. 75, 167-172 (2014)

45. Shtir, C. et al. Exome-based case-control association study using extreme phenotype design reveals novel candidates with protective effect in diabetic retinopathy. Hum. Genet 135, 193-200 (2016).

46. Rotimi, C. N. et al. In search of susceptibility genes for type 2 diabetes in West Africa: the design and results of the first phase of the AADM study. Ann. Epidemiol. 11, 51-58 (2001).

47. American Diabetes, A. Diagnosis and classification of diabetes mellitus. Diabetes Care 37, S81-90 (2014).

48. Rotimi, C. et al. Prevalence and determinants of diabetic retinopathy and cataracts in West African type 2 diabetes patients. Ethn. Dis. 13, S110-117 (2003).

49. McCarthy, S. et al. A reference panel of 64,976 haplotypes for genotype imputation. Nat. Genet. 48, 1279-1283 (2016).
50. Loh, P. R. et al. Reference-based phasing using the Haplotype Reference Consortium panel. Nat. Genet. 48, 1443-1448 (2016).

51. Durbin, R. Efficient haplotype matching and storage using the positional Burrows-Wheeler transform (PBWT). Bioinformatics 30, 1266-1272 (2014).

52. Zheng, X. et al. HIBAG - HLA genotype imputation with attribute bagging. Pharm J. 14, 192 (2013)

53. The Atherosclerosis Risk in Communities (ARIC) Study: design and objectives. The ARIC investigators. Am. J. Epidemiol. 129, 687-702 (1989).

54. Klein, R. et al. The association of atherosclerosis, vascular risk factors, and retinopathy in adults with diabetes: the atherosclerosis risk in communities study. Ophthalmology 109, 1225-1234 (2002).

55. Cheung, N. et al. Diabetic Retinopathy and the Risk of Coronary Heart Disease. Diabetes Care 30, 1742 (2007)

56. Yu, B. et al. Genome-wide association study of a heart failure related metabolomic profile among African Americans in the Atherosclerosis Risk in Communities (ARIC) study. Genet Epidemiol. 37, 840-845 (2013).

57. Willer, C. J., Li, Y. \& Abecasis, G. R. METAL: fast and efficient meta-analysis of genomewide association scans. Bioinformatics 26, 2190-2191 (2010).

58. Purcell, S. et al. PLINK: a tool set for whole-genome association and populationbased linkage analyses. Am. J. Hum. Genet 81, 559-575 (2007).

59. Zheng, X. et al. A high-performance computing toolset for relatedness and principal component analysis of SNP data. Bioinformatics 28, 3326-3328 (2012).

60. The Genomes Project, C. et al. A global reference for human genetic variation. Nature 526, 68 (2015).

(i) Open Access This article is licensed under a Creative Commons Attribution 4.0 International License, which permits use, sharing, adaptation, distribution and reproduction in any medium or format, as long as you give appropriate credit to the original author(s) and the source, provide a link to the Creative Commons license, and indicate if changes were made. The images or other third party material in this article are included in the article's Creative Commons license, unless indicated otherwise in a credit line to the material. If material is not included in the article's Creative Commons license and your intended use is not permitted by statutory regulation or exceeds the permitted use, you will need to obtain permission directly from the copyright holder. To view a copy of this license, visit http://creativecommons. org/licenses/by/4.0/.

This is a U.S. government work and not under copyright protection in the U.S.; foreign copyright protection may apply 2019 